\title{
Single molecule protein sequencing based on the superspecificity of tRNA synthetases
}

\author{
G. Sampath \\ P. B. 7849, J. P. Nagar P. O., Bengaluru-560078, India
}

\begin{abstract}
Single molecule de novo protein sequencing based on the 'superspecificity' of amino-acyl tRNA synthetases (aaRS) is proposed. An unfolded protein molecule is threaded through a nanopore in an electrolytic cell (e-cell) to expose the terminal residue in the e-cell's trans chamber. After the residue is cleaved with a peptidase, a set of tRNAs, their aaRSs, and ATP are added to trans. An aaRS charges a cognate tRNA molecule with the residue. The charged tRNA (along with the other reactants) is transferred to an extended e-cell with $\mathrm{N}(20 \leq \mathrm{N} \leq 61)$ pores in $\mathrm{N}$ individual cis chambers and a single trans chamber. Each pore holds an RNA molecule ending in a unique codon that is exposed in trans. The charged tRNA's anticodon base-pairs with the terminal codon of an RNA. If tRNAs and residues are fluorescently tagged with two different colors, the residue can be identified from the observed position of the color pair. As charging is 'superspecific' identification is unambiguous. The protein molecule in the first e-cell is advanced by one residue and the process repeated. In this approach there is no need for precise pore current or optical intensity measurements. Potential implementation issues are discussed. Several variations, including one in which the terminal residue is cleaved after charging, are also considered.
\end{abstract}

Keywords: protein sequencing; single molecule; nanopore; tRNA; amino acyl tRNA synthetase; codon; optical tag

\section{Introduction}

Biopolymer sequencing occupies a central place in the study of biological organisms and is usually focused on research or the diagnostic and therapeutic value of the information contained in a polymer (DNA, RNA, protein) sequence. DNA sequencing is now a mature area [1] and is accomplished by a number of technologies, starting with Sanger sequencing and proceeding to next generation techniques that make use of highly automated processes [2]. On the other hand protein sequencing is not as advanced, largely because there are 20 amino acids to work with, compared to four bases for DNA. It is currently done with one of the following methods: Edman degradation (ED), gel electrophoresis (GE), and mass spectrometry (MS) [3-5]. The current state of protein sequencing research is reviewed in [6].

More recently nanopores have been used to sequence DNA [7]. Briefly, an electrolytic cell [8] or e-cell consists of two chambers, named cis and trans, which contain an electrolyte, typically $\mathrm{NaCl}$ or $\mathrm{KCl}$, and are bridged by a thin membrane containing a nanopore. An electrical potential $\mathrm{V}$ applied between cis and trans ionizes the electrolyte and leads to current flow through the pore. With $\mathrm{KCl}$ as the electrolyte, $\mathrm{K}^{+}$ions flow toward the cathode and $\mathrm{Cl}^{-}$toward the anode. If a charged analyte molecule (DNA, RNA, or protein) is introduced into cis, it translocates through the pore in an appropriate direction. Both DNA and RNA carry a uniform negative charge along their backbone, so they flow from cis to trans in Figure 1 below. In contrast, seven of the standard 20 amino acids, namely D, E, K, R, H, C, and Y, carry a negative or positive charge whose value depends on the $\mathrm{pH}$ of the electrolyte [9]. This means that the net charge carried by a protein depends on the sequence of residues and also determines the direction of flow of the protein. Crucially, translocation of DNA, RNA, or protein through the pore causes a reduction in the pore current that varies with the volume of the monomer passing through the pore. This monomer-specific level of current blockade serves as the basis for current approaches to nanopore-based sequencing. Such methods are beginning to show promise and are set to compete with the more established methods mentioned above [10]. In contrast, nanopore-based protein sequencing is still in its infancy; a variety of methods, some in practice [11-14], others in theory [15-18], are known. Most recently it has been reported that 13 of the 20 standard amino acids (obtained by finely grinding a protein) riding on a carrier molecule while translocating through an aerolysin nanopore have long enough dwell times in the pore to allow discrimination among them based on the volume that each of them excludes in the pore [19]. This work is reminiscent of earlier theoretical work in which residues in a peptide are sequentially cleaved by an exopeptidase on the output side of the first of two pores in series (tandem pore) and then identified by the volume they exclude when they translocate through the second pore [15].

\subsection{The present work}

This report proposes a protein sequencing method in which the sequence of amino acids is obtained indirectly from a sequence of codons that code for the amino acids. An e-cell with a nanopore is used with an electrical potential (or other method, see Section 4) to hold the unfolded linear protein sequence in place while the terminal residue is exposed in trans. If the terminal residue is cleaved with an exopeptidase, and a set of tRNAs, their aminoacyl tRNA synthetases (aaRSs), and ATP are added to trans, a matching tRNA gets 'charged' with the residue by the corresponding aaRS. This charged tRNA (or aminoacyl tRNA) is transferred to a second modified e-cell with 
multiple nanopores each of which holds an RNA molecule ending in a codon coding for an amino acid. The anticodon in the tRNA binds with one of these RNA molecules by base-pairing with the terminal codon of the latter. With the tRNAs and residues fluorescently tagged [20] with two different colors, the occurrence of tRNA charging in the first e-cell and the identity of the residue in the second can both be detected optically. As the charging of a tRNA by a related aaRS is highly specific (the commonly used term is 'superspecific') [21-22], residue identification is unambiguous. The protein molecule in the first e-cell is advanced by one residue through the pore, and the process repeated until the protein has been sequenced. Several variations of this general scheme are also considered. In one of them a tRNA is charged with the exposed residue before it is cleaved. In this variation there are two versions, one with optical tagging and the other without.

This approach mimics the way mRNA is translated to protein in vivo but without involving the complexities present in the latter. E-cells with nanopores provide a controlled environment in which enzymes and processes similar to those that occur during translation can be used. Fluorescent tagging of residues and tRNAs enables the detection of salient events to drive the process in a controlled manner. Importantly precise measurements of optical intensities or nanopore currents are not necessary. Thus in the former case detection of fluorescent spots in the image is sufficient, in the latter case only the occurrence of a current blockade needs to be detected. Because no $a$ priori information about the protein is used the proposed method is capable of de novo sequencing.

\section{Designing a protein sequencing method based on the superspecificity of tRNAs}

The protein sequencing procedure that is the subject of this communication is motivated by the superspecificity property of aaRSs in a biological cell: a tRNA always gets charged by a related aaRS with the amino acid associated with it; error rates are on the order of 1 in 10000 [22].

Consider how translation of mRNA into protein occurs in vivo, that is, in a biological cell. This is a complex process that involves a host of enzymes and other factors interacting with codon-specific tRNAs to facilitate the synthesis of a peptide sequence. The following is a simplified description, see [23] for a detailed description of the dynamics of translation at the molecular structure level. peptide:

There are three major steps in translation, each of which results in an amino acid being added to a growing T1: The enzyme aaRS activates ATP and a cognate amino acid to form an amino acid AMP complex, charges the cognate tRNA with the amino acid at the latter's carboxyl end and releases the AMP, then releases the charged or aminoacyl tRNA. (This step is independent of translation: aminoacyl tRNAs are normally available in a cell for every one of the standard 20 amino acids for translation when the cell needs them.)

T2: An aminoacyl tRNA from Step T1 with anticodon matching the next codon along the mRNA binds with the latter by Chargaff base-pair matching.

T3: The aminoacyl tRNA from the residue previously attached to the growing peptide is cleaved and the incoming residue in an aminoacyl tRNA is attached to the latter. This last step is part of a reaction that involves the enzyme peptidyl transferase and several other factors.

Steps T2 and T3 take place in the ribosome, which consists of two subunits (named 40S and 60S in eukaryotes, and jointly named $80 \mathrm{~S}$ ), which together provide a platform on which mRNA and tRNAs are brought together to enable protein synthesis. The $30 \mathrm{~S}$ subunit is first bound to the mRNA. There are three tRNA binding sites in 80S: the A site holds an incoming aminoacyl-tRNA, the P site holds the tRNA attached to the last residue in the growing peptide (this tRNA is known as peptidyl-tRNA), and the E site provides an exit for tRNAs that have completed amino acid delivery. After an amino acid is added to the peptide, 80S moves along the mRNA to the next codon. Translation is error-free because tRNA charging is superspecific and base pairing of an anticodon with a matching codon is unambiguous. If any errors occur the enzymes involved uncharge the incorrect tRNA [21].

A protein sequencing procedure can be designed by borrowing and adapting elements of the above translation process. Consider the following three generic steps in protein sequencing, which are repeated for each successive residue in the protein:

G1: Hold the protein molecule in a fixed location where the terminal residue can be exposed and cleaved.

G2: Identify the residue.

G3: Advance the protein molecule to expose the next residue.

The three steps can be realized with the following three components $\mathrm{H} 1, \mathrm{H} 2$, and $\mathrm{H} 3$.

H1: An e-cell with a nanopore provides a platform for G1. The protein molecule is threaded through the pore and held in place by a voltage across the cis and trans chambers (and/or some other means, such as optical or magnetic tweezers, see Section 4) such that only the first residue is exposed in trans. An exopeptidase bound on the trans side to the membrane containing the pore (or, alternatively, free exopeptidase in solution) cleaves the exposed residue so it is free to bind to its cognate tRNA. 
H2: A cognate tRNA for the cleaved residue can be obtained by adding to trans a full set of tRNAs and the corresponding aaRSs, as well as ATP. This will result in one of the tRNAs being charged with the cleaved residue leading to an aminoacyl tRNA..

H3: The aminoacyl tRNA can be identified by base-pairing its anticodon with a codon in an RNA molecule. This requires a platform that holds a set of (at least) 20 RNA molecules, one (at least) for each of the 20 standard amino acids, ending in a unique triplet codon and held in a fixed location where the charged tRNA can base-pair with a matching codon. A setup similar to the one in $\mathrm{H} 1$ above can be used for this. It takes the form of an extended e-cell that has one trans chamber and 20 (or more) individual cis chambers and nanopores, one (or more) for a standard amino acid type, through which an RNA molecule is threaded 5' end first to expose a matching terminal codon in the common trans chamber. Identification can then be based on fluorescent tagging with two distinct colors, one for the tRNAs and the other for amino acids. In this case, the charged tRNA can be identified based on the two colors (which will be in close proximity) and their position in the extended e-cell. If the pores in the extended e-cell are sufficiently separated in space there is no possibility of a misread.
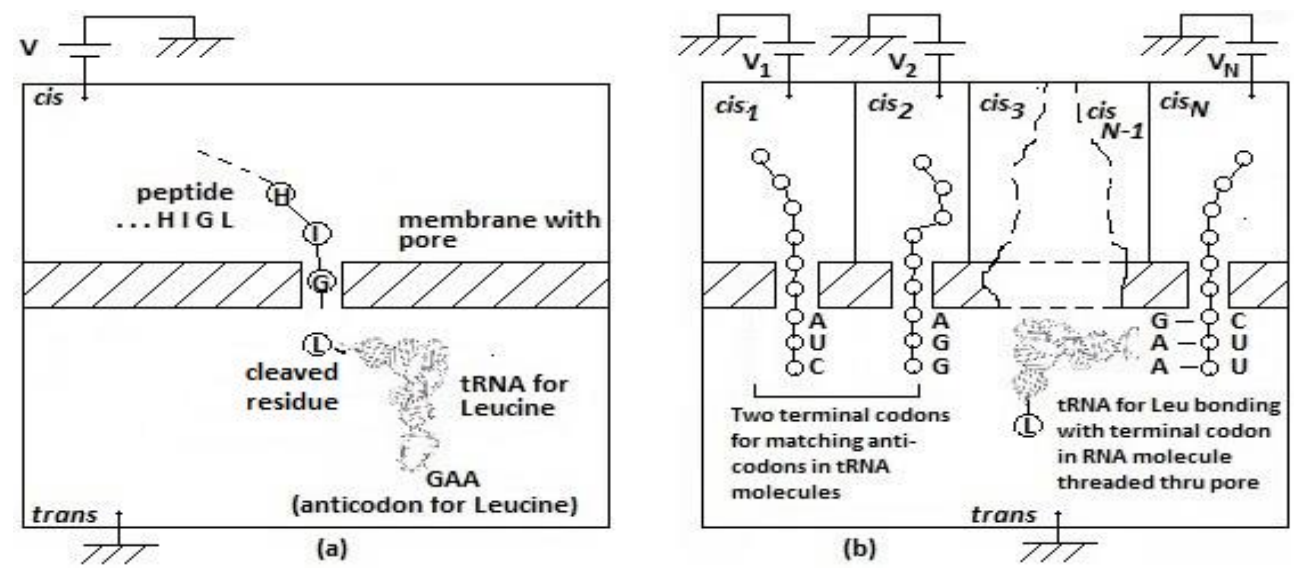

Fig 1. Electrolytic cells for sequencing a protein molecule (not to scale)

(a) Standard e-cell with cis and trans chambers. Voltage V provides electrophoretic force for analyte to translocate through pore such that first residue in protein is exposed and cleaved (residue is shown free) in trans by exopeptidase (not shown). Catalyzed by residue's cognate amino acyl tRNA synthetase and ATP, tRNA is charged at 3' end (terminal triplet CCA covalently binds with carboxyl terminal of residue). Occurrence of charging is detected optically with tags attached to residue and tRNA. All reactants in trans are then transferred to trans chamber of e-cell on the right.

(b) E-cell has $\mathrm{N}(20 \leq \mathrm{N} \leq 61)$ individual cis chambers each with pore through common membrane. trans chamber is common to all of them. Voltages $\mathrm{V}_{1}, \ldots, \mathrm{V}_{\mathrm{N}}$ are individually applied to $\mathrm{N}$ cis-trans pairs, independently control translocation of i-th RNA molecule through i-th pore. Each RNA molecule ends in unique codon that matches with anticodon in tRNA, tRNA in trans binds with some RNA by base-pairing. If residue and tRNAs are fluorescently tagged with two distinct colors $\mathrm{C}_{1}$ and $\mathrm{C}_{2}$, residue is identified from position of color pair $\mathrm{C}_{1}-\mathrm{C}_{2}$.

Figure 1 shows the two types of e-cells. In Figure 1(a) the terminal residue is L (Leucine); it is held in trans just outside the pore and then cleaved with an exopeptidase. (Only the cleaved residue is shown.) An aaRS charges a cognate tRNA with this free residue in trans. The charged tRNA is then transferred to the trans chamber of the second e-cell where its anticodon base-pairs with the terminal codon of an RNA molecule. The aminoacyl tRNA carries the anticodon GAA; the corresponding codon CUU codes for Leucine. In Figure 1(b) the codon CUU is the terminal triplet of the N-th RNA molecule. Residues are tagged with color $\mathrm{C}_{1}$, tRNAs with $\mathrm{C}_{2}$. After the color pair $\mathrm{C}_{1}-\mathrm{C}_{2}$ has been sensed and its position in trans (given by the number of the pore, 1 to $\mathrm{N}$ ) noted, the voltages $\mathrm{V}_{1}, \ldots$, $\mathrm{V}_{\mathrm{N}}$ can be set to suitable values to translocate all the RNA molecules (along with the base-paired tRNAs) fully into the trans chamber. Following this the contents of trans can be flushed out, the chamber refilled with electrolyte, and the pores re-threaded with RNA molecules as before for the next cycle.

The protein sequencing procedure is given next.

\section{Protein sequencing procedure}

The following sequence of steps is repeated for every residue in the target protein. The operations in the two e-cells 
can be pipelined for speedup.

\section{Identification procedure for a single residue}

Step 1. Attach fluorescent dye of some fixed color $\mathrm{C}_{1}$ to every residue in analyte protein molecule, color $\mathrm{C}_{2}$ to each of $\mathrm{N}(20 \leq \mathrm{N} \leq 61)$ tRNAs.

Step 2. Advance protein molecule through pore in first e-cell (Figure 1(a)) with voltage V (or some other means, see below) set such that the first residue (C-terminal or $\mathrm{N}$-terminal) remains stationary outside pore in trans chamber while rest of polymer remains in pore and cis.

Step 3. Cleave residue with an exopeptidase (amino peptidase for $\mathrm{N}$-terminal, carboxy peptidase for C-terminal), which may be bonded to membrane on trans side or free in trans solution of first e-cell.

Step 4. Add full set of tRNA molecules to trans of first e-cell, corresponding aaRSs, and ATP so 3' binding site (CCA terminus) of some tRNA attaches to cleaved residue (Leucine in example above). All other tRNAs remain in trans solution. Charged tRNA will be recognizable from tandem color pair $\mathrm{C}_{1}-\mathrm{C}_{2}$ as it diffuses around in solution. All other tRNAs will be associated only with color $\mathrm{C}_{2}$ and can be so distinguished.

Step 5. Prepare second e-cell (Figure 1(b)). Thread RNA molecule ending in unique triplet corresponding to codon for amino acid $i$ through pore i. Set voltages $V_{i}$ such that terminal triplet in RNA molecule is in trans and trailing bases are in pore $\mathrm{i}$ and $c i s_{\mathrm{i}}$. In each case pore current is lower than free pore current because of blockading effect of RNA molecule in pore.

Step 6. Transfer all tRNAs (including charged one) and other reactants (including aaRSs) in solution out of trans of first e-cell into trans of second e-cell. Charged tRNA's anticodon base-pairs with terminal codon of one of the RNAs, say $\mathrm{RNA}_{\mathrm{i}}$. $\mathrm{C}_{1}-\mathrm{C}_{2}$ will remain in close vicinity of $\mathrm{RNA}_{\mathrm{i}}$.

Step 7. Mark position of color pair $\mathrm{C}_{1}-\mathrm{C}_{2}$ as pore number i. Other tRNAs could have their anticodons base-paired with terminal codons of other RNAs and will therefore remain in vicinity of those RNAs. Remaining tRNAs will be moving around in trans. If pores in second e-cell are widely separated there is no possibility of confusing color pair $\mathrm{C}_{1}-\mathrm{C}_{2}$ in position $\mathrm{i}$ with color $\mathrm{C}_{2}$ of any other tRNA base-paired with an RNA or any floating tRNA. Record $i$ as identity of residue.

Step 8. Set all $\mathrm{V}_{\mathrm{i}}$ to appropriate values so that all threaded RNA molecules translocate fully into trans carrying with them any tRNAs that may have base-paired with their anticodons. This results in end of current blockade in all $\mathrm{N}$ pores, signaling end of read.

Step 9. Flush out trans chamber of second e-cell and refill with electrolyte.

This approach may be compared with recent methods for partial or full peptide sequencing. (With partial sequences the goal is usually identification of the peptide's parent protein.) In [13] the protein molecule is broken into short peptides and the carboxyl end of each peptide covalently attached to a glass slide. Optical tags attached to selected residue types are detected to identify the terminal residue at the N-end, following which N-end ED is used to cleave that residue. Tagging of a small number ( 2 or 3 ) of amino acid types is sufficient to identify the parent protein. In a slightly different approach, a theoretical model is described in [24] where peptides ending in a Cysteine (Cys) residue are assumed bound to a glass slide, and the binding times of various optically tagged sensor molecules known as N-terminal amino acid binders (NAABs) to the N-terminal residue of the peptide are calculated. The results suggest that by using multiple weak binders in succession a terminal residue may be identified at a rate > 97\% [24]. Effectively this could lead to the full protein sequence being known, so an implementation of this approach is not limited to protein identification.

In contrast with [13] and [24], the method proposed here is aimed at protein sequencing (rather than identification). Sequencing may be done from the $\mathrm{C}$-terminal or the $\mathrm{N}$-terminal end of an unfolded protein molecule. A tRNA that carries the anticodon for the terminal residue attaches itself to the C-terminal end of the freed residue to charge the latter. Because charging is highly specific, multiple rounds of binding (as required in [24], to increase the probability of a correct read) and optical intensity measurements are not necessary.

\section{Potential implementation issues}

The proposed scheme is considerably simpler than the in vivo translation process. The latter is dynamically regulated by a number of processes occurring simultaneously in a restricted and crowded space. In contrast the targets in the present case, namely the cleaved residue in the first cell and the terminal codons in the N RNA molecules in the second cell, can be targeted in a more controlled manner. As with in vivo translation the superspecificity of aaRSs makes residue identification highly accurate.

In the discussion that follows four assumptions are made for the procedure in Section 3:1) the terminal residue is exposed in trans while all the successor residues are in the pore and cis; 2) the protein is held stationary under the 
influence of the voltage V set to an appropriate value (or by some other means, discussed below); 3) the exposed residue is cleaved by a free exopeptidase in the trans solution; and 4) a tRNA synthetase charges the residue with a cognate tRNA. The rest of this section looks at potential implementation of such a scheme and discusses potential problems that may arise therefrom as well as their potential solutions. There are broadly three issues to consider. 1) residue cleaving; 2) polymer position control; and 3) event detection.

\subsection{Cleaving of a terminal residue}

Cleaving of the exposed residue of the shrinking protein molecule is central to the proposed sequencing procedure. Proteolysis is a widely studied process and there is a vast literature on the subject; comprehensive reference works are available [25,26]. In the present context the objective is limited to cleaving the first exposed residue of a protein molecule in the pore of the first e-cell. Some applicable methods are discussed elsewhere [15] in the context of nanopore-based protein sequencing using a tandem electrolytic cell. In particular see [27] and [28] for information on cleaving at the $\mathrm{C}$ - and $\mathrm{N}$-terminal with carboxypeptidases and aminopeptidases respectively. Recently an enzyme designed on a computer and named Edmanase that cleaves residues at the N-end of a protein has been described [29]. Naturally occurring proteins that recognize specific N-terminal amino acids have been studied for possible use in peptide sequencing, leading to variants of the adapter protein ClpS that can recognize $\mathrm{N}$ terminal F (Phenylalanine) and W (Tryptophan) [30].

In the procedure in Section 3, exposure in trans was limited to the terminal residue. The reason for this is to prevent spurious cleaving at an internal bond. Such targeted cleaving can be done either by an exopeptidase that is covalently bonded to the membrane (or pore) on the trans side or free exopeptidase in the trans solution. However as noted below this requires very precise control of the molecule by one of several ways discussed below. Such precision can be avoided if spurious cleavage does not occur, so exposure need not be limited to the terminal residue. This is discussed in the next subsection.

\subsection{Polymer position control}

The analyte molecule (protein in the first e-cell, RNAs in the second) translocates rapidly through a pore, which makes it difficult to measure the change in the current blockade level due to individual monomers [31]. Currently available detector bandwidths do not support such measurements. A range of methods to slow down the polymer in its passage through the nanopore have been reported, an early review can be found in [32] (but also see [33]). More recent work can be found in [34-40]. The primary aim in all of the above slowdown methods is to allow discrimination of bases in DNA and residues in proteins (and, in one case, unfolding of the protein as well [34]).

In the present case, however, there is no need to make precise blockade current measurements, it suffices to detect the occurrence of a blockade, as the goal is to hold the protein in the pore in the first e-cell and the RNA molecules in their pores in the second e-cell such that only the terminal residue and the terminal codons are exposed, while the protein or RNA remains stationary. One possible way to achieve stationarity of the polymer is to balance the electrophoretic force due to the voltage against the motive force due to diffusion. In principle this may be easier to do with DNA and RNA, which carry a uniform negative charge along the backbone, whereas most proteins carry a weak net charge that may be positive or negative. In the latter case better control may be possible by attaching a trailing homopolymer polyZ where $\mathrm{Z}$ is a charged amino acid with appropriate polarity. However, as noted in [32] this is somewhat difficult because the diffusive force is spread throughout the length of the polymer both inside the pore and outside and it is hard to have stop-and-go control over the length of the polymer segment that is inside the pore. There are multiple ways to resolve this problem: a) mechanical position control, b) hydraulic position control, and c) tethering the molecule to some fixed location. They may be used singly or in combination, along with the electrical potential $\mathrm{V}$ across cis and trans.

\section{a) Mechanical control}

Mechanical approaches provide a viable way to hold the polymer in place with little movement. Optical or magnetic tweezers [41-43] are particularly effective. Here the electrophoretic force on a polymer can be exactly balanced by a mechanical force, resulting in the polymer remaining stationary, with nanometer to sub-nanometer precision. In [41,42] optical tweezers hold a double stranded DNA molecule for insertion into a nanopore with sub-nanometer positioning precision, thus allowing for base-level discrimination. Alternatively a polymer can be attached to a colloidal bead held between the poles of a magnet, leading to controlled translocation of the polymer through the pore [43]. The magnetic field counteracts the usual electric potential to precisely retract the molecule after its entry into the pore. In principle, with these methods an unfolded protein can be linearly stretched out fully so that stop- 
and-go control can be exercised over its translocation through the pore.

\section{b) Hydraulic control}

The use of hydraulic pressure to control the movement of a polymer through a pore was first studied in [44]. This approach has been investigated in detail recently [45]. With pressures in the range 0.5 to 2 atmospheric pressures (atm) applied with a plunger mounted in the cis or trans chamber to act against the electric field over the length of the pore, blockade current changes on the order of 5-10 nA have been measured with the analyte trapped for considerably long periods of time. (In comparison, in most nanopore experiments the currents measured are around 100-200 pA [8].)

\section{c) Chemical tethering}

Covalent bonding makes it possible to hold one end of a molecule in position. In [13] and [24] this is done to identify residues in peptides via total internal reflection fluorescence (TIRF) microscopy. In [46] a DNA strand is bound to the vestibule of a biological pore, in [47] to an adapter attached to an alpha hemolysin pore.

In the present case, all three approaches are suitable. For example, similar to [13] and [24] a peptide can be tethered to a glass slide in the cis chamber of the first e-cell, then drawn into a nanopore with an electric field. With a sufficiently large field the peptide can be stretched fully through the pore, and the glass slide controlled with a plunger in cis to which hydraulic pressure may be applied to insert/retract the peptide into/from the pore. This enables the controlled exposure of only the terminal residue in the trans chamber while the rest of the tethered molecule remains in the pore and cis. A similar setup can be used with optical or magnetic tweezers.

With the second e-cell the RNA molecules can be controlled with tweezers or tethered to the membrane in the respective cis chamber near the pore entrance on the cis side (see Figure 1(b)). In the latter case to ensure correct base pairing of the anticodon on a tRNA with the terminal codon of an RNA molecule in the second e-cell an RNA oligo should have $\mathrm{k}+3$ bases where $\mathrm{k} *$ length of base $\approx$ length of pore such that only the terminal triplet is exposed in trans. Once again with a suitable $\mathrm{V}_{\mathrm{i}}>0$ the $\mathrm{i}$-th RNA molecule, which carries a uniform negative charge, is drawn into the the $\mathrm{i}$-th pore and remains fully stretched inside while only the terminal triplet is exposed. However, the length of the pore places a rigid constraint on the value of $\mathrm{k}$. One possible solution is to use a tethered DNA molecule with a dangling single strand 3-base extension consisting of only the triplet codon (or its equivalent, since in DNA base T replaces base $\mathrm{U}$ ).

\subsection{Event detection}

Four salient events occur in sequence in the procedure in Section 3, the times of their occurrence are instrumental in driving the identification procedure. These are

a) cleaving of the exposed residue in the trans chamber of the first e-cell;

b) charging of a tRNA with the cleaved residue;

c) transfer of the charged tRNA to the second cell; and

d) binding of the transferred tRNA in the second e-cell to the terminal codon of some RNA molecule.

With optical tags attached all four events are easy to detect. With color $C_{1}$ associated with residues in the protein molecule and color $\mathrm{C}_{2}$ with tRNAs, event a is flagged by color $\mathrm{C}_{1}$. This generates a moving spot in the image as the free residue diffuses around in the trans chamber of the first e-cell. Following this the addition of tRNAs to trans causes a number of moving spots of color $\mathrm{C}_{2}$. When a cognate tRNA attaches itself to the residue, the $\mathrm{C}_{1}$ and $\mathrm{C}_{2}$ spots form a $\mathrm{C}_{1}-\mathrm{C}_{2}$ pair, this flags the occurrence of event $\mathrm{b}$. When the transfer in c occurs, it leads to a single $\mathrm{C}_{1}-\mathrm{C}_{2}$ pair and a bunch of $\mathrm{C}_{2}$ spots due to the uncharged tRNAs in the trans chamber of the second e-cell, all of them diffusing around in trans. When the charged tRNA binds with one of the RNAs by base-pairing of the anticodon in the former with the terminal codon of the latter the $\mathrm{C}_{1}-\mathrm{C}_{2}$ pair location become more or less fixed. This flags event $d$. (Some of the uncharged tRNAs may base-pair with the other RNAs thereby fixing some of the $\mathrm{C}_{2}$ spots while the $\mathrm{C}_{2}$ spots of the remaining uncharged tRNAs will continue to move around. None of this affects residue identification.)

\subsection{Relaxing the constraint on residue or terminal codon exposure}

The condition that only the terminal residue be exposed in the trans chamber of the first e-cell can be relaxed if an exopeptidase that reliably cleaves only the terminal residue is used. In this case part or all of the target peptide can be allowed into trans while the part of the molecule that is in the pore remains stationary. This provides more flexibility in polymer position control. In this case if a poly-X trailer of length $\mathrm{m}$, where $\mathrm{X}$ is one of the charged residues (D, E, K, R, H, C, Y), is attached to the tail of the target peptide the peptide can be paid out into trans one 
residue at a time into trans with one of the control methods discussed above.

Thus let the peptide sequence $\mathrm{R}_{1} \mathrm{R}_{2} \ldots \mathrm{R}_{\mathrm{n}}$ going from $\mathrm{N}$-terminal to C-terminal (or vice versa) be extended by a poly-X trailer of length $\mathrm{m}$, where $\mathrm{X}$ is one of the charged residues $(\mathrm{D}, \mathrm{E}, \mathrm{K}, \mathrm{R}, \mathrm{H}, \mathrm{C}, \mathrm{Y})$ and $\mathrm{m}$ *length of residue $\mathrm{X}$ $>$ length of the pore in the first electrolytic cell. Let $X$ be the negatively charged residue D. If $X_{1}$ is tethered to the membrane near the pore entrance in cis, then with $\mathrm{V}$ set to a large enough positive value (see Figure 1(a)) the extended molecule D D ... $D_{m} R_{1} R_{2} \ldots R_{n}$ is drawn into the pore and remains fully stretched through the pore. Although two or more residues from the entry end of $R_{1} R_{2} \ldots R_{n}$ are now exposed in trans a tRNA can attach itself only to $R_{n}$ because this is where the carboxyl end is available for charging. In successive cycles $R_{n}, R_{n-1}, \ldots, R_{1}$ get attached to a corresponding tRNA, each of these Rs being cleaved at the end of a cycle and exposing the next R's carboxyl end for charging. Any of the above position control methods, tweezer, hydraulic, or tethering can be used to ensure that the analyte remains close to the pore on the trans side so that charging and cleaving events occur in a known small neighborhood where they can be detected fairly easily with optical tagging.

\section{Discussion}

1) In the procedure in Section 3 it was assumed that the terminal residue is cleaved before a tRNA is charged with it. This prevents any potential steric clashes with the pore or the membrane during charging. However, if it can be determined experimentally that a tRNA can be charged with the terminal residue before the latter is cleaved from the protein, some distinct advantages can accrue. First, as noted in Section 4, the use of optical/magnetic tweezers or tethering to control the position of the peptide becomes simpler. Second, if the charging time is known (it can be determined experimentally) then there is no need for optical tags at all. Third, because the tRNA is bound to the exposed terminal residue, following charging all other reactants can be flushed out of the trans chamber of the e-cell before cleaving of the residue. Following this the charged tRNA can be transferred to the second e-cell (along with any free peptidase used for cleaving). Since there are no other reactants, this is the only transfusion into the second e-cell. The charged tRNA binds to the anticodon of one of the RNAs by base-pairing. As in the case of charging, prior knowledge of base-pairing times can be used to determine when the next step, which is that of identifying the residue, using optical or non-optical means, can take place.

Nevertheless optical tags are useful to precisely determine the times when charging occurs in the first e-cell and when base-pairing of the tRNA with an RNA occurs in the second e-cell. Thus if the tRNAs are optically tagged with a single color $\mathrm{C}_{2}$ the occurrence of charging can be detected when the $\mathrm{C}_{2}$ of the charged tRNA spot is bound to the terminal residue and remains stationary (all other $\mathrm{C}_{2}$ spots will be diffusing around in the e-cell. Since these other reactants are flushed out, these moving $\mathrm{C}_{2}$ spots will disappear, leaving only the fixed $\mathrm{C}_{2}$ of the charged tRNA. After cleaving, when the tagged tRNA is transferred to the second e-cell, the event of base-pairing of its anticodon to the codon of an RNA (RNAi) is flagged by the $\mathrm{C}_{2}$ spot becoming stationary in the vicinity of pore $\mathrm{i}$. The spatial position of $\mathrm{C}_{2}$ in the trans chamber of the second e-cell is sufficient to identify the residue.

Based on the above discussion, an alternative residue identification procedure is given next, it is repeated for every residue in the target protein. As with the procedure in Section 3, the two e-cells can be pipelined.

\section{Alternative identification procedure for a single residue}

Step A1. Attach fluorescent dye of some fixed color color $\mathrm{C}_{2}$ to each of $\mathrm{N}(20 \leq \mathrm{N} \leq 61)$ tRNAs. (This step is optional.)

Step A2. Same as Step 2 in Section 3 except that protein molecule enters pore C-terminal first.

Step A3. Same as Step 4 in Section 3. Some tRNA gets charged with exposed uncleaved residue. If optical tagging is done as in Step A1, this leads to stationary $\mathrm{C}_{2}$ spot near exit of pore in trans, and moving $\mathrm{C}_{2}$ spots due to all uncharged tRNAs.

Step A4. Flush these remaining tRNAs (and all other reactants) out of trans while refilling with electrolyte. If optical tagging is done only $\mathrm{C}_{2}$ spot due to charged tRNA remains in trans of e-cell 1.

Step A5. Same as Step 5 in Section 3.

Step A6. Cleave residue with a carboxyl peptidase. If optical tagging is used in Step A1, C2 spot due to charged tRNA will start diffusing around in trans.

Step A7. Transfer charged tRNA from trans of e-cell 1 to trans of e-cell 2.

If optical tagging is used in Step A1, $\mathrm{C}_{2}$ spot due to charged tRNA will start diffusing around in trans of e-cell 2. When anticodon in tRNA base-pairs with terminal codon of $\mathrm{RNA}_{i} \mathrm{C}_{2}$ will remain stationary near $\mathrm{RNA}_{i}$; residue is identified by pore number I; go to Step A9.

Step A8. (Optical tagging not used) Set voltages $V_{i}$ in second e-cell to values such that all the RNAs retract into their cis chambers, except the one with whose terminal codon charged tRNA has base-paired. In latter case current through pore is still at blockade level, in all other cases current blockade ceases. Number of 
blockaded pore identifies residue.

Step A9. Flush out trans chamber of second e-cell and refill with electrolyte. Rethread RNA molecules through all $\mathrm{N}$ pores as before with terminal codon exposed. (Alternatively with optical tagging base-paired tRNAs can be detached from RNAs with appropriate enzyme before flushing, in which case rethreading is not needed.)

The procedure can be further simplified if tRNA and aaRS pairs are added individually in sequence until charging occurs. Writing the standard set of amino acids as $\mathbf{A A}=[\mathrm{G}, \mathrm{A}, \mathrm{S}, \mathrm{C}, \mathrm{D}, \mathrm{T}, \mathrm{N}, \mathrm{P}, \mathrm{V}, \mathrm{E}, \mathrm{Q}, \mathrm{H}, \mathrm{M}, \mathrm{I}, \mathrm{L}, \mathrm{K}, \mathrm{R}$, $\mathrm{F}, \mathrm{Y}, \mathrm{W}]$, a maximum of 20 cycles, one per amino acid, would be required. The residue may be identified in the first cycle (as G, Glycine) in the best case and in the $20^{\text {th }}$ cycle (as W, Tryptophan) in the worst case, with an average of 10 cycles (for E, Glutamic Acid). In this case only the first electrolytic cell is needed if optical tags are used (only the tRNA needs to be tagged). The residue (with the attached tRNA) is cleaved, followed by flushing of trans and refilling with electrolyte. Without optical tags, the contents of trans in the first e-cell are transferred to trans in the second e-cell, where the tRNA with attached residue base-pairs with one of the RNAs. Following this the voltages $\mathrm{V}_{\mathrm{i}}$ are set to appropriate values so that all RNAs except the one that has base-paired with the transferred tRNA retract into their cis chambers thus unblocking their nanopores. The excepted pore remains blockaded, this identifies the residue. The trans chamber of the second e-cell is then flushed and refilled with electrolyte.

If charging of a tRNA with the exposed residue before cleaving is problematic then the possibility of modifying aaRSs to enable such charging can be considered. Such modification may be designed similar to [48], which has lists of the primary sequences of redesigned tRNA synthetases for use as NAABs in protein sequencing.

2) Most of the voltage drop in an electrolytic cell is across the pore [8]. The drop in the cis and trans chambers is only $\sim 2 \%$ so the motive force in these chambers is mostly diffusion. Because of this an analyte molecule may have to be drawn into the pore by some agency such as dielectrophoretic trapping [49]. With proteins a possible solution is to use a sodium dodecyl sulphate (SDS) sheath to give the protein a uniform negative charge that gets stripped out as the protein is drawn into the pore [12,50].

3) In the case when charging is done before cleaving, the protein molecule must enter the pore in the first e-cell carboxyl end first as the tRNA attaches to this end. To ensure entry at the correct end the amino end can be capped with a small molecule of a size large enough that it cannot enter the pore. This also requires a polyZ trailer of length $\mathrm{b}$, where $\mathrm{Z}$ is a selected amino acid and $\mathrm{b}^{*}($ length of $\mathrm{Z})>$ length of pore. Similarly an RNA molecule in the extended e-cell has to enter a pore at the 3 ' end. Once again capping can be used for this. An alternative approach in both cases would be to reverse the roles of cis and trans, which can effectively be achieved by reversing the voltage polarity. Entry into a pore at the wrong end can be detected optically or when the desired event (charging of tRNA, binding of tRNA to codon) does not occur within some expected time.

4) Nanopores play a secondary role in the proposed approach. Their only purpose is to linearize and keep unfolded a protein/RNA molecule such that only the terminal residue or codon is exposed to chemical reactions, which, in the case of protein, minimizes the possibility of an exopeptidase acting like an endopeptidase and cleaving the protein internally. Thus such properties as pore length (or equivalently membrane thickness), pore diameter, electro-osmotic effects due to charged residues on the inner wall of the pore lumen, and similar properties that affect conventional nanopore-based sequencing are not important. The only exception to these considerations is that the pore diameter be sufficiently small that the protein does not fold inside the pore and that residues get exposed in trans one at a time in sequence order.

5) The procedure in Section 3 can also be used for sequencing a protein in the bulk, similar to C-terminal protein sequencing by digestion with serine carboxypeptidase [27] or N-terminal sequencing with an aminopeptidase [28]. Thus multiple copies of a protein from a sample can be subjected at the $\mathrm{N}$-terminal or C-terminal to cleaving with an amino or carboxyl exopeptidase. The resulting free residues can then be charged with tRNAs by successively inserting each type of tRNA, ATP, and the corresponding synthetase into the solution. This insertion loop is prematurely exited when the occurrence of charging is observed optically as noted earlier. The reactants are flushed out and the process repeated for successive terminal residues in the remaining molecule.

\section{References}

[1] J. M. Heather and B. Chain. "The sequence of sequencers: the history of sequencing DNA". Genomics 107, 1-8, 2016.

[2] E. Pettersson, J. Lundeberg, and A. Ahmadian. "Generations of sequencing technologies". Genomics 93, 105-11, 2009. doi:10.1016/j.ygeno.2008.10.003

[3] R. J. Simpson. Proteins and Proteomics: A Laboratory Manual, CSHL Press, 2008.

[4] E. de Hoffmann and V. Stroobant. Mass Spectrometry: Principles and Applications, 3rd edn., Wiley, 2007.

[5] T. Rabilloud and C. Lelong. "Two-dimensional gel electrophoresis in proteomics: a tutorial". J. Proteomics 74, 1829-1841, 
2011.

[6] N. Callahan, J. Tullman, Z. Kelman, and J. Marino. "Strategies for development of a next-generation protein sequencing platform". Trends Biochem. Sci. 2019. doi:10.1016/j.tibs.2019.09.005.

[7] H. Bayley. "Nanopore sequencing: from imagination to reality". Clin. Chem. 61, 25-31, 2015.

[8] J. E. Reiner, A. Balijepalli, J. W. F. Robertson, J. Campbell, J. Suehle, and J. J. Kasianowicz. "Disease detection and management via single nanopore-based sensors". Chem. Rev. 112, 6431-6451, 2012.

[9] D. L. Nelson and M. M. Cox. Lehninger's Principles of Biochemistry, $4^{\text {th }}$ edn., W H Freeman, 2005.

[10] D. Deamer, M. Akeson, and D. Branton. "Three decades of nanopore sequencing". Nature Biotechnol. 34, 518-524, 2016.

[11] Y. Zhao, B. Ashcroft, P. Zhang, H. Liu, S. Sen, W. Song, J. Im, B. Gyarfas, S. Manna, S. Biswas, C. Borges, and S. Lindsay. "Single-molecule spectroscopy of amino acids and peptides by recognition tunneling". Nature Nanotechnol. 9, 466-473, 2014.

[12] E. Kennedy, Z. Dong, C. Tennant, and G. Timp. "Reading the primary structure of a protein with $0.07 \mathrm{~nm}^{3}$ resolution using a subnanometre-diameter pore". Nature Nanotechnol. 11, 968-976, 2016.

[13] J. Swaminathan, A. A. Boulgakov, E. T. Hernandez, A. M. Bardo, J. L. Bachman, J. Marotta, A. M. Johnson, E. V. Anslyn, and E. M. Marcotte. "Highly parallel single-molecule identification of proteins in zeptomole-scale mixtures". Nature Biotechnol. 36, 1076-1082, .

[14] L. Restrepo-Pérez, C. Joo, and C. Dekker. "Paving the way to single-molecule protein sequencing". Nature Nanotechnology 13, 786-796, 2018. https://doi.org/10.1038/s41565-018-0236-6

[15] G. Sampath. "Amino acid discrimination in a nanopore and the feasibility of sequencing peptides with a tandem cell and exopeptidase”. RSC Adv. 5, 30694-30700, 2015.

[16] J. Swaminathan, A. A. Boulgakov, and E. M. Marcotte. "A theoretical justification for single molecule peptide sequencing". PLOS Comput. Biol. 11, e1004080, 2015.

[17] P. Boynton and M. Di Ventra. "Sequencing proteins with transverse ionic transport in nanochannels". Sci. Rep. 6, 25232, 2016.

[18] J. Wilson, L. Sloman, Z. He, and A. Aksimentiev. "Graphene nanopores for protein sequencing". Adv. Funct. Mater. 26, 4830-4838, 2016.

[19] H. Ouldali, K. Sarthak, T. Ensslen, F. Piguet, P. Manivet, J. Pelta, J. C. Behrends, A. Aksimentiev, and A. Oukhaled. "Electrical recognition of the twenty proteinogenic amino acids using an aerolysin nanopore". Nature Biotech., December 16, 2019.

[20] C. Joo, H. Balci, Y. Ishitsuka, C. Buranachai, and T. Ha. "Advances in single-molecule fluorescence methods for molecular biology". Annu. Rev. Biochem. 77, 51-76, 2008.

[21] O. O. Favorova. "Superspecificity of aminoacyl-tRNA-synthases". Mol. Biol. (Mosk). 18, 205-226, 1984.

[22] D. Goodsell. "Aminoacyl-tRNA Synthetases". PDB Molecule of the Month, April 2001. doi:10.2210/rcsb_pdb/mom_2001_4

[23] T. A. Steitz. "A structural understanding of the dynamic ribosome machine". Nature Reviews 9, 242-253, 2008.

[24] S. Rodriques, A. Marblestone, and E. Boyden. "A theoretical analysis of single molecule protein sequencing via weak binding spectra". PloS One 14, e0212868, 2019. doi: 10.1371/journal.pone.0212868

[25] A. J. Barrett, N. D. Rawlings, and J. F. Woessner. (eds.) Handbook of Proteolytic Enzymes, Academic Press, 1998.

[26] D. L. Crimmins, S. M. Mische, and N. D. Denslow. "Chemical cleavage of proteins on membranes". Curr Protoc Protein Sci., 2001. doi: 10.1002/0471140864.ps1105 s19

[27] K. Breddam and M. Ottesen, "Determination of c-terminal sequences by digestion with serine carboxypeptidases: the influence of enzyme specificity". Carlsberg Res. Commun. 52, 55-63, 1987.

[28] A. Taylor, “Aminopeptidases: structure and function". FASEB J. 7, 290-298, 1993.

[29] B. Borgo and J. J. Havranek. "Computer-aided design of a catalyst for Edman degradation utilizing substrate-assisted catalysis". Protein Sci. 24, 571-579, 2015.

[30] J. Tullman, N. Callahan, B. Ellington, Z. Kelman, and J. P. Marino. "Engineering ClpS for selective and enhanced Nterminal amino acid binding". Appl. Microbiol. and Biotechnol. 103, 2621-2633, 2019.

[31] S. Carson and M. Wanunu. "Challenges in DNA motion control and sequence readout using nanopore devices". Nanotech. 26, 074004, 2015.

[32] U. F. Keyser. "Controlling molecular transport through nanopores". J. R. Soc. Interface 8, $1369-1378,2011$. doi:10.1098/rsif.2011.0222

[33] C. Plesa, S. W. Kowalczyk, R. Zinsmeester, A. Y. Grosberg, Y. Rabin, and C. Dekker. "Fast translocation of proteins through solid state nanopores". Nano Lett. 13, 658-663, 2013. dx.doi.org/10.1021/nl3042678

[34] J. Nivala, D. B. Marks, and M. Akeson. "Unfoldase-mediated protein translocation through an alpha-hemolysin nanopore". Nat Biotechnol. 31, 247-250, 2013.

[35] L. Mereuta, M. Roy, A. Asandei, J. K. Lee, Y. Park, I. Andricioaei, and T. Luchian. "Slowing down single-molecule trafficking through a protein nanopore reveals intermediates for peptide translocation". Sci. Rep. 4, 3885, 2014. doi: 10.1038/srep03885

[36] S. W. Kowalczyk, D. B. Wells, A. Aksimentiev, and C. Dekker. "Slowing down DNA Translocation through a Nanopore in Lithium Chloride". Nano Lett. 12, 1038-1044,2012. dx.doi.org/10.1021/nl204273h

[37] Z. Tang, Z. Liang, B. Lu, J. Li, R. Hu, Q. Zhao, D. Yu. "Gel mesh as "brake" to slow down DNA translocation through solid-state nanopores". Nanoscale 7, 13207-13214, 2015.

[38] F. Cecconi, M. A. Shahzad, U. M. B. Marconi, and A. Vulpiani. "Frequency-control of protein translocation across an 
oscillating nanopore". Phys.Chem.Chem.Phys. 19, 11260, 2017. DOI:10.1039/c6cp08156h

[39] A. Asandei, I. S. Dragomir, G. Di Muccio, M. Chinappi, Y. Park, and T. Luchian. "Single-molecule dynamics and discrimination between hydrophilic and hydrophobic amino acids in peptides, through controllable, stepwise translocation across nanopores". Polymers, 10, 885, 2018. doi:10.3390/polym10080885

[40] X. Liu, Y.Zhang, R. Nagel, W. Reisner, and W. B. Dunbar. "Controlling DNA tug-of-war in a dual nanopore device". arXiv:1811.11105v1 [physics.bio-ph], 27 Nov 2018.

[41] U. F. Keyser, J. van der Does J, C. Dekker, and N. H. Dekker. "Optical tweezers for force measurements on DNA in nanopores". Rev. Sci. Instrum. 77, 105105, 2006. doi:10.1063/1.2358705.

[42] E. H. Trepagnier, A. Radenovic, D. Sivak, P. Geissler, and J. Liphardt. "Controlling DNA capture and propagation through artificial nanopores". Nano Lett. 7, 2824-2830, 2007.

[43] H. Peng and X. S. Ling. "Reverse DNA translocation through a solid-state nanopore by magnetic tweezers". Nanotech. 20, 185101, 2009. doi:10.1088/0957-4484/20/18/185101

[44] B. Lu, D. P. Hoogerheide, Q. Zhao, H. Zhang, Z. Tang, D. Yu, and J. A. Golovchenko. "Pressure-controlled motion of single polymers through solid-state nanopores". Nano Lett. 13, 3048-3052, 2013.

[45] H. Zhang, Q. Chen, Y. Wu, Y. Wang, X. Bei, and L. Xiao. "The temporal resolution and single-molecule manipulation of a solid-state nanopore by pressure and voltage". Nanotech. 29, 495501, 2018. doi:/10.1088/1361-6528/aae190

[46] S. Howorka and H. Bayley. "Probing distance and electrical potential within a protein pore with tethered DNA". Biophys. J. 83, 3202-3210, 2002.

[47] J. Clarke, H. C. Wu, L. Jayasinghe, A. Patel, S. Reid, and H. Bayley H. "Continuous base identification for single-molecule nanopore DNA sequencing". Nature Nanotech. 4, 265-70, 2009.

[48] J. J. Havranek and B. Borgo. "Molecules and methods for iterative polypeptide analysis and processing", 2013 U. S. Patent US20140273004A1. Patent Assignee: Washington University in St Louis.

[49] K. J. Freedman, L. M. Otto, A. P. Ivanov, A. Barik, S.-H. Oh, and J. B. Edel. "Nanopore sensing at ultra-low concentrations using single-molecule dielectrophoretic trapping". Nature Commun. 7, 10217, 2016. doi: 10.1038/ncomms 10217

[50] L. Restrepo-Pérez, S. John, A. Aksimentiev, C. Joo, and C. Dekker. "SDS-assisted protein transport through solid-state nanopores". Nanoscale 9, 11685-11693, 2017.

Email: sampath_2068@yahoo.com 